\title{
Multifaceted bundle interventions shown effective in reducing VAP rates in our multidisciplinary ICUs
}

\author{
Abdel Latif Marini, Raymond Khan, Shihab Mundekkadan
}

Ministry of National Guards, Health Affairs

\begin{abstract}
Ventilator associated pneumonia (VAP) remains a worldwide harm associated with hospital acquired infection. Our VAP rate at King Abdulaziz Medical City was 4.0 per 1000 patient days at baseline. All regulatory bodies continue to emphasize the importance of reducing these infections and include a guideline of practice recommendations to address them, notably the VAP bundle by the Institute for Healthcare Improvement. Our baseline compliance was low and measured to be $83 \%$; this was perceived as an opportunity to work on revising our interventions in the ICUs. An improvement team gathered in 2013, and following the "model of improvement" methodology, along with a sequence of parallel PDSAs, they were able to increase compliance with the care bundle and sustain it above $95 \%$ for more than one year. This translated in a decrease in the VAP rate from 4.0 to 0.8 in all different multidisciplinary ICUs.
\end{abstract}

\section{Problem}

King Abdulaziz Medical City (KAMC) in Riyadh, Saudi Arabia, was established by a royal decree in May 1983. With a bed capacity of 1 220 operational beds, KAMC is a leading medical centre, accredited by Joint Commission International, and provides all types of care to National Guard personnel, their dependents, and other eligible patients, starting from primary health care up to tertiary specialized care. The facility includes 41 wards, including surgical and medical wards, obstetrics and gynaecology, and oncology. The facility also has a cardiac and liver centre, along with 13 different critical care units serving adults and paediatrics, 14 major operating suites, emergency care and trauma centres, ambulatory (outpatient) care, dental, and long term care/rehabilitation.

The VAP (ventilator acquired pneumonia) rate at KAMC was averaging 4.0 per 1000 patient days between 2012 and 2013. The hospital administration considered VAP as a "big dot" quality indicator, and hence was perceived as an opportunity to revise and improve our interventions for VAP prevention in the ICUs.

The Ministry of National Guards Health Affairs at KAMC partnered with the Institute for Healthcare Improvement (IHI) in 2012 to build capacity in the science of improvement, and launch several improvement projects. It was observed that all previous interventions to improve the care for VAP patients were isolated, and not embedded into the system. Despite improvements in VAP rates, we wanted to achieve a systemic approach that would lead to sustainable change as we aimed for the target of zero VAP.

A multidisciplinary VAP team was created with an aim to improve compliance with the VAP bundle, and subsequently reduce VAP rates. Our population included all ICUs: neurology (eight beds), intermediate medical ICU (14 beds), trauma (eight beds), surgical (nine beds), burns (eight beds), and general ICU (21 beds), a total of 68 beds. VAP was defined according to the National Healthcare Safety Network criteria.[1]

\section{Background}

Evidence based clinical practice guidelines have recommended multiple strategies to reduce VAP. Despite this, VAP remains a significant cause of mortality and morbidity in mechanically ventilated patients worldwide.[2,3] Acquiring VAP is associated with greater use of broad spectrum antibiotics,[4] prolonged time spent on a mechanical ventilator,[5] and increased ICU and hospital length of stay,[2] ultimately costing more than US $\$ 40000$ per episode.[6]

All regulatory bodies and infection prevention organisations emphasise the importance of reducing hospital acquired infections, and have published guidelines of practice recommendations to address them, notably the VAP bundle by the IHI. This IHI ventilator bundle, a grouping of best practices that, when applied together, may result in substantially greater improvement, has been implemented in many ICUs, along with teamwork and communication strategies such as structured multidisciplinary rounds and daily goal setting, to wean and remove patients from ventilators as quickly as possible while providing evidence based care.

\section{Baseline measurement}

Our VAP rate was measured by the infection control department (total number of VAP cases $/$ (ventilator days $\times 1000)=$ VAP rate per 1000 patient days). Our baseline VAP rate before starting the project was 4.0 despite several earlier solo initiatives. The bundle compliance is measured as per the below formula:

\begin{abstract}
- Numerator: the number of intensive care unit patients on mechanical ventilation at the time of survey, for whom all four elements of the ventilator bundle are documented and in place
\end{abstract}

- Denominator: total number of intensive care unit patients on 
mechanical ventilation

Our baseline VAP rate before implementing the project interventions was averaging 4.0 per 1000 patient days between 2012 and 2013, with a bundle compliance averaging $83 \%$.

For more information see appendix 1: the diagram shows the relationships between the aim of our project and the changes to be tested and implemented.

See supplementary file: ds6680.jpg - "Appendix 1: Relationship between the aim of our project, and the changes to be tested and implemented."

\section{Design}

A multidisciplinary team was initiated in October 2012, and included a physician leader, infection control practitioner, nurses, respiratory therapist (RT), and performance improvement specialist. Our team members were all trained about the science of improvement, and were coached by quality professionals to write the project charter and develop the driver diagram along with primary and secondary drivers, and choose the change ideas.

After thorough review of the literature, the team agreed on a modified IHI VAP prevention care bundle. The ventilator care bundle was a group of evidence based practices that, when implemented together for all patients on mechanical ventilation, result in dramatic reductions in the incidence of VAP. Our bundle included:

1. Elevation of the head of the bed between $30^{\circ}$ and $45^{\circ}$

2. Daily "sedation vacations" and assessment of readiness to extubate

3. Peptic ulcer disease prophylaxis

4. Deep venous thrombosis prophylaxis

5. Daily oral care with chlorhexidine

6. Endotracheal tube with inline and subglottic suctioning

7. Cuff pressure between $25 \mathrm{~cm}$ to $30 \mathrm{~cm} \mathrm{H} 2 \mathrm{O}$.

\section{Strategy}

We used the model of improvement to conduct this project. Several strategies were tested and implemented, including engagement in a collaborative program with the $\mathrm{IHI}$, the formation of a critical care safety team, audits of physician practice, incorporating endotracheal cuff pressure monitoring, implementation of a new oral care protocol, and the introduction of endotracheal tubes and tracheostomy with subglottic suctioning.

Many PDSA cycles were conducted, sometimes in parallel to accelerate learning. A data measurement sheet was tested on paper. This data sheet was then tabulated in Excel to yield compliance with the bundle whenever nurses input their data, by choosing from a drop down menu (Yes/No/NA). This automated sheet made it easy for the nurses in each unit to know their preliminary bundle compliance level at bed side each month.
Measurement of bundle compliance was done by front line users, including nurses and clinical nurse specialists as per the following guideline, with variability in time of assessment between day and night shifts, and including weekends:

ICU: Two to three times per week for 60 observations per month

IMCU: Three times per week for 60 observations per month

TICU, SICU, and NCCU: Three times per week; $100 \%$ of ventilated patients per month

The entire bundle was considered compliant only if all seven items were compliant, unless contraindicated. The data retrieved were analysed for accuracy and validity by quality management, tabulated in run charts, and shared monthly with the VAP team, comprising all ICU nurse managers and front line staff. The below is a summary of our PDSA that addressed different elements of the VAP bundle.

Process 1. Head of bed elevation (HOB)

We have tested processes and system changes that allowed us to improve performance on elevation of head of the bed. Some of these changes were:

- Adding HOB documentation to the nursing flow sheet. Nurses were required to document $\mathrm{HOB}$ every four hours to the ICU nursing flow sheets

- Empower respiratory therapists (RTs) to notify nursing if the head of the bed is not elevated; alternatively, place the patient in this position with nursing assistance

- Including this intervention as a default order in ICU admission order sets. Exceptions to this were: log-roll protocol, pelvic fractures, morbid obesity, prone position, intra-aortic balloon pump, and unstable spine not cleared by neurosurgery

This element was measured by the VAP data collectors, from direct observation of the HOB of applicable patients.

Process 2. Daily sedation vacations

We have tested process changes that allowed us to improve performance on daily sedation vacations, and daily assessment of readiness to extubate. Some of these changes were:

- Implementing a protocol to lighten sedation daily at 8 am (for patients who met the criteria as per the protocol), to assess for readiness to extubate.

- Include precautions to prevent self-extubation, such as increased monitoring and vigilance during the trial

- Post compliance with the intervention in the ICU hallway, to encourage change and motivate staff. 
This element of the bundle was measured by looking at the medication administration records for the sedation being withheld, and confirming with the nurse.

Daily assessment of readiness for extubation:

A ventilator weaning protocol was tested in our ICUs. It allowed the $R T s$ to wean all applicable mechanically ventilated patients starting at $9 \mathrm{am}$, one hour after sedation is held. The criteria for the weaning trial included the patient being awake and off sedation for at least one hour, being haemodynamically stable, on $\mathrm{FiO} 2<50 \%$, and positive end-expiratory pressure (PEEP) $<8 \mathrm{~cm} \mathrm{H} 20$. This element was assessed for compliance by looking at the ICU flow sheet and the RTs' notes.

Process 3 \& 4. Peptic ulcer disease prophylaxis, and deep vein thrombosis (DVT) prophylaxis

We implemented a force function in this process, which allowed us to improve performance on peptic ulcer disease and DVT prophylaxis. The main intervention was to include peptic ulcer disease prophylaxis and DVT prophylaxis as part of our ICU order admission order set. All ICU patients had to have DVT prophylaxis, either by mechanical measure, ie thromboembolic deterrent (TED) stockings and a sequential compression device, or a pharmacological measure (heparin or enoxaparin). Moreover, these were included as items for discussion on ICU daily multidisciplinary rounds. Compliance with this bundle element was measured by checking the documentation for any form of DVT prophylaxis in the chart, and checking the patient for a non-pharmacological intervention.

\section{Process 5. Oral care protocol}

We developed a nursing protocol for oral care using a unified oral kit package. This allowed us to standardise the process among nurses in our ICUs, and allowed utilisation of evidence based practices using chlorhexidine solution. The protocol mandated nurses to perform oral care every four hours by sponge swabs soaked in $0.12 \%$ chlorhexidine gluconate solution, and using the brush every 12 hours. Nurses received competencies about the new protocol, and the practice was reinforced by the clinical resource nurse. This element of the bundle was measured by reviewing the documentation in the chart, equipment utilisation reports, and periodical evaluation of the patients' oral cavities.

\section{Process 6. Cuff pressure monitoring}

The cuff pressure was maintained between 20 to $30 \mathrm{~cm} \mathrm{H20}$. It was measured by RTs with a VBM cuff pressure gauge, and recorded every six hours. Shortage of these cuff pressure tools affected the compliance of this measure. The team made sure to procure additional cuff pressure devices to cover the large ICU population. It is worth mentioning that we found our Trauma ICU population had a higher risk of VAP, so a continuous cuff pressure control device was used for these patients.

Process 7. Subglottic suctioning
All of our patients in ICU had a closed inline suctioning system. All trauma patients and ICU patients who were anticipated to require mechanical ventilation for more than 48 hours were required to be intubated with oral evacuation endotracheal (ET) tubes (tubes that allow subglottic secretion drainage). This change was difficult to accomplish, as we continued to receive patients from emergency room with the regular ET tube. Stocking the appropriate tube in emergency carts was done across the hospital, and also in the rapid response team intubation kit. Patients with subglottic ET tubes were connected to the wall suction at a set negative pressure (100 $\mathrm{mmHg}$ with a 20 second interval and duration of 8 seconds).

For all of these interventions, the previous and current compliance with the VAP bundle was shared with the ICU leadership and displayed in public in the ICU hallway, to encourage change and motivate staff. See appendix 2: "Individual unit compliance with the VAP bundle" for more information.

See supplementary file: ds7106.png - "Appendix 2: Individual unit compliance with the VAP bundle"

\section{Results}

Our data provided evidence that the initiation of a multidisciplinary VAP prevention team, using an adapted $\mathrm{IHI}$ care bundle approach with the science of improvement methodology, lead to a sustained reduction in the incidence of VAP in our ICUs.

The HOB elevation and sedation vacation bundle elements were initially the most deficient (94\%), but they improved greatly by the end of the project (99\%).

Improvement took place over time. We used run charts to report our compliance over time and detect any non-random variation. A remarkable drop in VAP rate was noted, as the bundle compliance kept rising above $95 \%$. In our project, the bundle compliance increased from 83\% to $97 \%$ between May 2013 and June 2014. Additionally, the VAP rate decreased from a baseline average of 4.0 to 0.8 . The results were deemed sustainable, as the compliance of the bundle was above the median for more than six data points, creating a shift (non-random variation). Translating these results into occurrences of VAP episodes, the number dropped from 32 per year in 2013 to four episodes per year in 2014.

The NHS sustainability model was used as a diagnostic tool that helped identify strengths and weaknesses in our implementation plan.[7] The team members scored the elements under each factor of the model (process, staff, and organisation). This exercise helped the project members to predict the likelihood of sustainability for our project, and to divert resources into the areas that need more attention. In our case, it was the "senior leadership support" and "staff training to sustain the process" factors that required attention.[7] See appendix 3 for the run chart showing the VAP rate compared to VAP bundle compliance over time / data collection tool / NHS sustainability scoring.

A cost effectiveness study was not performed for this project; however, we can estimate VAP cost from previous literature and 
multiply the cost by the rate change we observed, to approximate the saving in health care cost. Considering that each episode of VAP costs approximately USD $\$ 40000,[6]$ and our VAP rate changed from 4 to 2 per 1000 ventilator days, our saving would be USD $\$ 80000$ for the period of the project.

See supplementary file: ds7214.pptx - "Appendix 3: Results"

\section{Lessons and limitations}

The strengths of our project include the large number of patients, approximately 4000 , treated in our ICUs over one year. Another important strength is the measurement of our primary outcome (VAP incidence) by an experienced, unchanging infection control team, and having compliance data validated by a quality management specialist.

There are several lessons we learned from this project; firstly, the greatest barrier to the implementation of the bundle was the reluctance of consultants to use the sedation vacation protocol. There were fears of unplanned extubation and desaturation due to agitation and anxiety. Secondly, we learned that a multidisciplinary team approach to ventilator care is essential to the success of the project. All of the stakeholders in the process must be included, in order to gain the buy-in and cooperation of all parties. Thirdly, some of the strategies that we utilised to attract and retain excellent team members included using data to define and solve the problem, reporting to the steering committee, finding champions within the hospital who were of sufficiently high profile to lend the effort immediate credibility, and working with those who wanted to work on the project, rather than trying to convince those who did not. In order to enlist support and engage staff, it was important to share baseline data on VAP rates, share the results of improvement efforts with front line staff, and celebrate success by having VAP days or VAP gatherings. The data was limited by the fact that, although our ICUs consist of 68 beds, this study is from a single centre. Further, we adapted the $\mathrm{IHI}$ bundle to suit our local characteristics and dynamics, which may not be applicable to other healthcare systems. Finally, despite being able to maintain a high level of bundle compliance during the project, it was only measured for one year.

\section{Conclusion}

As supported by the literature, implementing a multifaceted intervention was effective in significantly reducing the VAP rates in our critical care patient population. Compliance was achieved by incorporating the bundle into daily multidisciplinary rounds, and through regular audit and feedback. To sustain the improvement, a sustainability plan was drafted to establish policy and procedure, and make sure that the infrastructure would keep the change intervention in place and ongoing. To support the success of your project, it should be endorsed by science, led by stakeholders, guided by measures, and supported by management.
1. Guidelines for the management of adults with hospitalacquired, ventilator-associated, and healthcare-associated pneumonia. Am J Respir Crit Care Med 2005;171(4):388-416.

2. Chahoud J, Semaan A, Almoosa KF. Ventilator-associated events prevention, learning lessons from the past: $A$ systematic review. Heart Lung 2015;44(3):251-9.

3. Melsen WG, Rovers MM, Groenwold RH, et al. Attributable mortality of ventilator-associated pneumonia: a metaanalysis of individual patient data from randomised prevention studies. Lancet Infect Dis 2013;13(8):665-71.

4. Hayashi $\mathrm{Y}$, Morisawa K, Klompas M, et al. Toward improved surveillance: the impact of ventilator-associated complications on length of stay and antibiotic use in patients in intensive care units. Clin Infect Dis. 2013;56(4):471-7.

5. Rello J, Ollendorf DA, Oster G, et al. Epidemiology and outcomes of ventilator-associated pneumonia in a large US database. Chest 2002;122(6):2115-21.

6. Warren DK, Shukla SJ, Olsen MA, et al. Outcome and attributable cost of ventilator-associated pneumonia among intensive care unit patients in a suburban medical center. Crit Care Med 2003;31(5):1312-7.

7. NHS Institute for Innovation and Improvement. Sustainability model and guide. http://www.qihub.scot.nhs.uk/media/162236/sustainability m odel.pdf

\section{Declaration of interests}

Nothing to declare.

\section{Acknowledgements}

The intensive care, quality management, and infection control departments, King Abdulaziz Medical City, Ministry of National Guards Health Affairs, Riyadh, Saudi Arabia.

\section{Ethical approval}

According to the policy activities that constitute research at King Abdul Aziz Medical City, this work met criteria for operational improvement activities exempt from ethics review. Local policies and procedure do not require IRB approval for improvement projects of that kind.

The work is primarily intended to improve local care, not provide generalisable knowledge in a field of inquiry. Ethical approval was not obtained as this is considered to be a quality improvement project, where all VAP bundle interventions were validated by evidence based research, and testing was performed solely for improvement purposes.

\section{References}

